\title{
Influence of Oxidation Degree of Graphene Oxide on Its Nuclear Relaxivity and Contrast in MRI
}

\author{
Zinia Mohanta,* Sumana K. Gaonkar, Manoj Kumar, Jitender Saini, Vivek Tiwari, Chandan Srivastava, \\ and Hanudatta S. Atreya
}

Cite This: ACS Omega 2020, 5, 22131-22139

Read Online

\section{ACCESS \\ Џlll Metrics \& More \\ Article Recommendations \\ Supporting Information}

ABSTRACT: Graphene oxide (GO) serves as a versatile platform for various applications, with the oxygen content of GO playing an important role in governing its properties. In the present study, different GO types covering a wide range of oxidation degree were prepared using our newly developed two-step method involving ball milling of graphite followed by its oxidation to GO. In addition to the variations in their physicochemical properties, the different GO types exhibited differences in proton relaxivity due to their paramagnetic nature. Nuclear magnetic resonance spectroscopy studies showed that the degree of oxidation of GO perturbs its nuclear relaxation properties and, together with intercalated $\mathrm{Mn}^{2+}$ ions, provides large contrast variation in magnetic resonance imaging (MRI). The study for the first time reveals that the surface chemistry of GO affects its relaxivity and opens up new avenues for developing tunable GO-based contrast agents in magnetic resonance imaging for diagnostics and therapies.

\section{INTRODUCTION}

Graphene oxide (GO) serves as a versatile platform owing to its hydrophilicity, high aspect ratio, and high degree of functionalization capability. ${ }^{1,2}$ The oxygen content of GO plays an important role in determining its physical and chemical properties such as photoluminescence, adsorption, $\zeta$-potential, solubility, electrical conductivity, and thermal conductivity. ${ }^{3-5}$ The degree of oxidation of GO is also important for determining its toxicity and interaction with biomolecules. ${ }^{6,7}$ The focus, therefore, has been on development of methods for modulating the degree of oxidation of GO, which can be achieved by either modification of the oxidation conditions of graphite or reduction of as-synthesized graphene oxide. ${ }^{8,9}$

Among its various applications, GO has been used as a substrate for loading agents to improve contrast in magnetic resonance imaging (MRI), ${ }^{10-12}$ a widely used technique for clinical diagnostics and imaging of soft tissue. ${ }^{13,14}$ Using nonionizing radiation, MRI provides high spatial resolution, noninvasiveness, and real-time visualization that aides in the detection of tumors, cysts, microfractures, and anomalies in various parts of the body. ${ }^{15-17}$ However, smaller lesion size and subtle changes in contrast property limit proper identification and diagnosis of the disease. ${ }^{18,19}$ For enhanced sensitivity to detect subtle changes, exogenous contrast agents are injected. ${ }^{20,21}$

Our objective was to develop appropriately functionalized GO as a contrast agent for MRI applications. The contrast ability depends on the paramagnetic behavior of GO, which affects its nuclear relaxation property, termed as "relaxivity."
Currently, the role of GO in the enhancement of proton relaxivity remains unclear. Previous studies have shown that GO exhibits paramagnetic behavior due to the presence of paramagnetic impurities such as $\mathrm{Mn}^{2+}$ ions; ${ }^{23}$ these metal ions are entrapped between graphite sheets during the potassium permanganate-based oxidative procedure used for producing GO. ${ }^{24}$ In the present study, different GO types covering a wide range of oxidation degree were prepared using a two-step method. ${ }^{5}$ On probing the effect of GO on proton relaxivity using nuclear magnetic resonance (NMR) spectroscopy in combination with other techniques, we observed that GO with different degrees of oxidation exhibited varied relaxivity effects. It was found that the effect of GO on water proton relaxation depends on both the $\mathrm{Mn}^{2+}$-ion concentration and the degree of oxidation. The two factors together help in varying the contrast of images in MRI, which is required for accurate diagnosis. The approach, thus, for the first time provides a method to combine two parameters and helps in designing appropriately oxidized GO having a desired relaxivity for MRI applications.

Received: May 13, 2020

Accepted: August 14, 2020

Published: August 27, 2020 


\section{RESULTS AND DISCUSSION}

2.1. Characterization of the Synthesized GO. The morphology of GO was studied using atomic force microscopy (AFM). Due to ball milling, the precursor undergoes morphological changes and various defects are introduced during the process. ${ }^{25}$ During the initial hours of ball milling, shearing and breaking of the graphite particles occur. This phenomenon can be observed till $30 \mathrm{~h}$ of ball milling (under conditions of our setup). On further ball milling, these small particles start sintering and forming larger sheets, also known as cold welding. ${ }^{26,27}$ All of the graphites were ball-milled separately to get ball-milled graphitic precursors. On oxidizing these precursors, we get the corresponding GO. Such variations in morphology of the GO are revealed in particle thickness measured by AFM, as shown in Figure 1 (the AFM

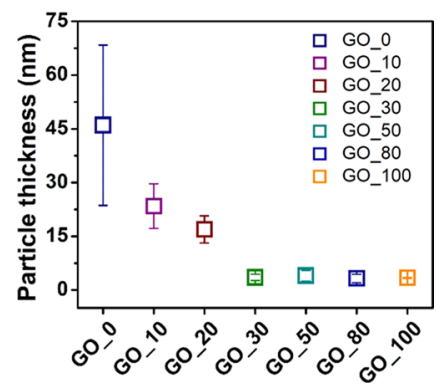

Figure 1. Particle thickness of GO samples obtained using AFM.

images are shown in Figure S1 of the Supporting Information). GO_0 was highly polydispersive and had the highest thickness among all GO. The sheet size, layer thickness, and dispersity decreased till GO_30. GO_50 had a larger sheet size, indicating that $\mathrm{GO} \_50$ consists of sintered particles of BMG 50. GO 80 and GO 100 had much more structured morphology due to sintering process. The particle thickness was found to decrease along the GO series (Figure 1; the values are tabulated in Table S1).

The oxidation degree is calculated from: (i) the ratio of reacted graphite (GO) to the total product (unreacted graphite and GO), obtained by integrating the appropriate peaks in ssNMR spectra (Figure S2) and (ii) the ratio of nonaromatic carbon to total carbon obtained from the deconvolution of high-resolution C 1s X-ray photoelectron spectra (XPS) (Figure S3). The oxidation degree of the GO in Figure 2a indicates an overall increase in the oxidation degree along the GO series. The $\mathrm{Fe}^{3+} / \mathrm{Fe}^{2+}$ ion was initially expected to be present due to contamination from the ball milling jar, but on atomic absorption spectroscopic investigation, no trace of such contamination was observed. To measure the $\zeta$-potential, which is an indirect measure for colloidal stability, electro- phoretic light scattering (ELS) was used. The $\zeta$-potential values for each GO are tabulated in Table S2 and plotted in Figure $2 \mathrm{~b}$. Similar to the oxidation degree, the $\zeta$-potential increases along the GO series.

The density of defects for GO $\left(I_{\mathrm{D}} / I_{\mathrm{G}}\right)$ shown in Figure $2 \mathrm{c}$ was calculated by deconvolution of the Raman spectra (Figure S4). The Raman band at $1350 \mathrm{~cm}^{-1}$ arises from $A_{1 g}$ and disorder-induced mode and is assigned as the $\mathrm{D}$ band; the bands at 1532 and $1598 \mathrm{~cm}^{-1}$ are a combination of $A_{1 g}, E_{1 g}$, and $E_{2 g}$ vibration modes and are assigned to the $G$ band and amorphous carbon, respectively. The intensities of the $\mathrm{D}$ and $\mathrm{G}$ bands were used to calculate the defect density, and the results are tabulated in Table S3 and plotted in Figure 2c. The defect density, while decreasing along the GO series, was not found to follow any particular pattern.

Graphene oxide exhibits paramagnetic behavior. This has been attributed to the presence of $\mathrm{Mn}^{2+}$ ions in GO. ${ }^{23,24}$ The intercalated $\mathrm{Mn}^{2+}$ ions exist in GO due to the use of potassium permanganate $\left(\mathrm{KMnO}_{4}\right)$ for oxidation of graphitic precursor to obtain GO. The $\mathrm{Mn}^{2+}$ concentration is measured using electron paramagnetic resonance (EPR) spectroscopy. The $\mathrm{Mn}^{2+}$-ion concentration (Figure $3 \mathrm{~b}$ ) was calculated from the spins obtained from the EPR spectra (Figure 3a). From Figure $3 \mathrm{~b}$, we observe that $\mathrm{GO} 80$ contains a higher amount of $\mathrm{Mn}^{2+}$ ions and that $\mathrm{GO} 550$ has a low amount of $\mathrm{Mn}^{2+}$. Notably, GO_0 has a much higher amount of $\mathrm{Mn}^{2+}$ than $\mathrm{GO} 100$, yet GO_100 exhibits better relaxivity than GO_0. This implies that there are other factors involved in governing the relaxation behavior of GO.

No peak for Mn lattice was detected in XRD; this indicates that the $\mathrm{Mn}$ is not present in any polycrystalline phase. ${ }^{28}$ The observation of a well-resolved hyperfine structure (Figure 3a) indicates that $\mathrm{Mn}^{2+}$ ions exist in the compound as quite magnetically diluted paramagnetic complexes rather than in the form of some impurities of concentrated Mn salts. ${ }^{24,29}$ The large hyperfine coupling is an indicator of the octahedral coordination of the Mn species. The narrow singlet line at the center of the EPR spectra can be attributed to carbon radicals.

2.2. Correlation of Oxidation Degree and Relaxivity in GO. We plotted defect density, $\zeta$-potential, and $\mathrm{Mn}^{2+}$ concentration against oxidation degree to investigate the degree of correlation among them. On linearly fitting the plots, as shown in Figure 4, we found that " $\zeta$-potential vs oxidation degree" and "defect density vs oxidation degree" were found to have Pearson's correlation coefficient greater than 0.8 , while the $\mathrm{Mn}^{2+}$ concentration vs oxidation degree plot had the correlation coefficient value of 0.52 , indicating that $\mathrm{Mn}^{2+}$ concentration and oxidation degree are mutually exclusive to each other and are independent factors. The contribution of these two factors, $\mathrm{Mn}^{2+}$ concentration and
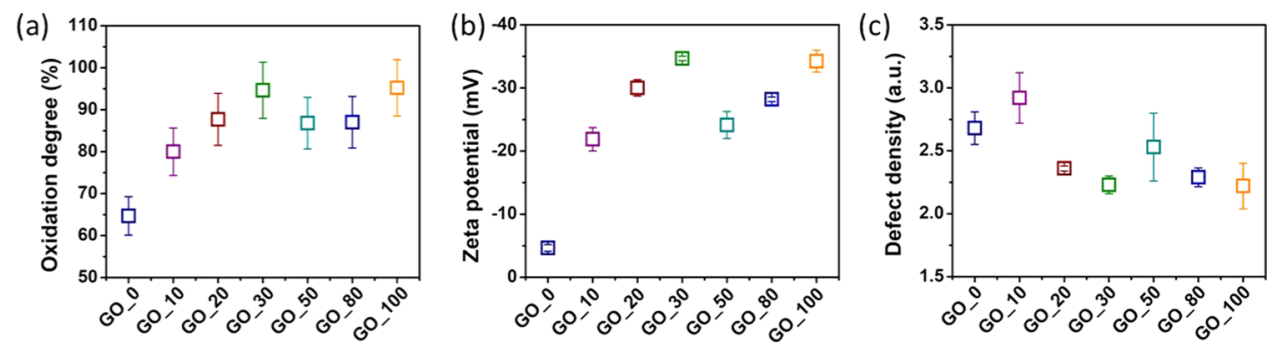

Figure 2. $(\mathrm{a}-\mathrm{c})$ Variations in oxidation degree, $\zeta$-potential, and defect density along the GO series. 

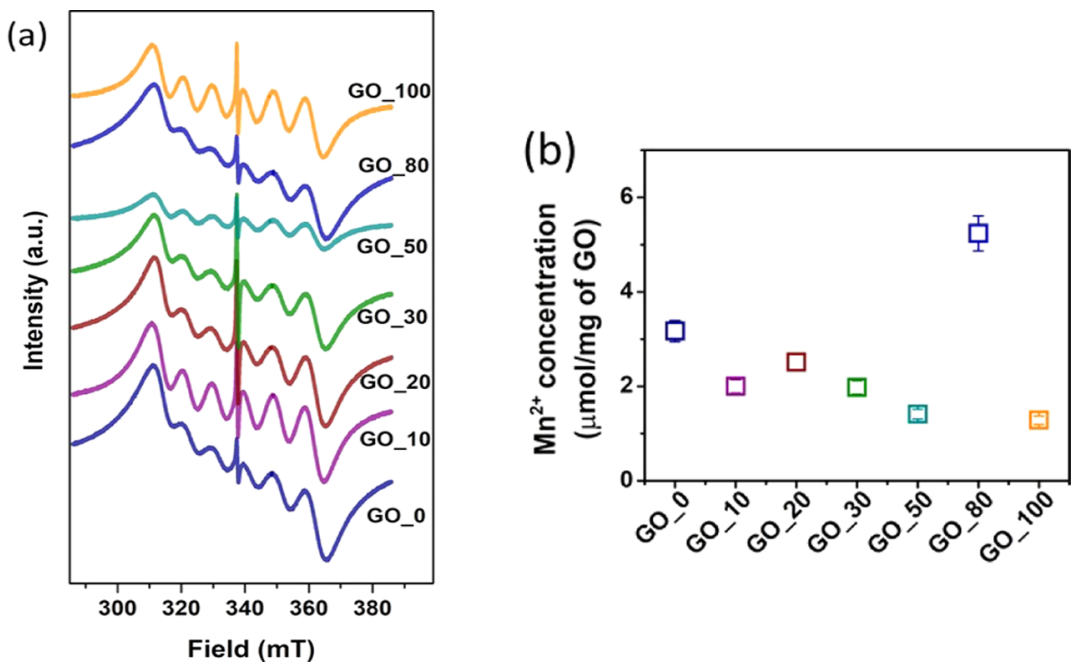

Figure 3. (a) EPR spectra recorded at $298 \mathrm{~K}$ of all GO showing six hyperfine lines at an operating frequency of $9.45 \mathrm{GHz}$ and field band of $286-$ $386 \mathrm{mT}$. (b) Variation of $\mathrm{Mn}^{2+}$ concentration along the GO series.
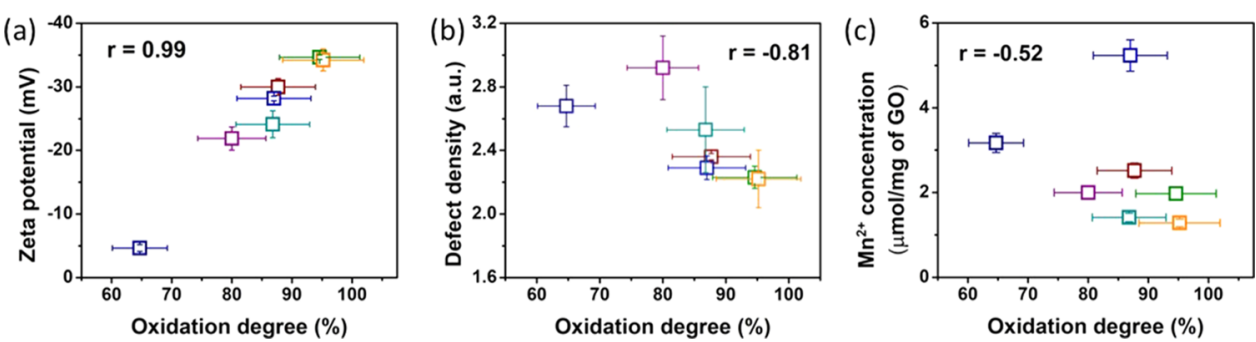

Figure 4. Correlation of oxidation degree of GO with (a) $\zeta$-potential, (b) defect density, and (c) $\mathrm{Mn}^{2+}$ concentration and estimation of Pearson's $\mathrm{r}$ (correlation coefficient) value. Color scheme of GO: blue square, GO_0; purple square, GO_10; red square, GO_20; green square, GO_30; peacock-green square, GO_50; blue square, GO_80; yellow square, $\mathrm{GO}_{-} 100$.
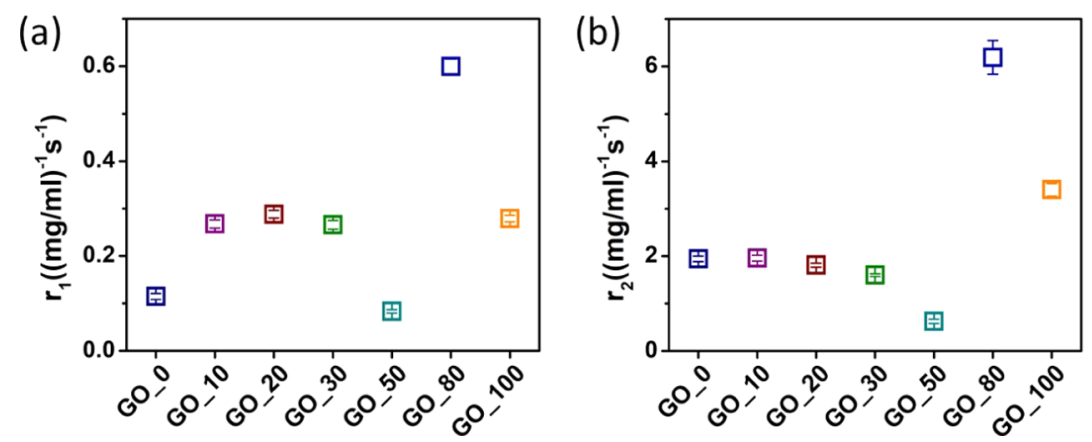

Figure 5. (a, b) Measured $r_{1}$ and $r_{2}$ relaxivities of different GOs. $T_{1}$ and $T_{2}$ measurements were carried out at a magnetic field strength of $9.4 \mathrm{~T}$ at a temperature of $298 \mathrm{~K}$.

oxidation degree, to the relaxation behavior of $\mathrm{GO}$ was further explored.

Paramagnetic materials alter the nuclear spin relaxation $\left(T_{1}\right.$ and $T_{2}$ ) of surrounding water protons, thereby facilitating a good image contrast for accurate diagnosis in magnetic resonance imaging. ${ }^{30}$ The potency of such contrast agents termed as "relaxivity" is quantitatively measured by its effect on relaxation rates of the water protons. It is measured as the change in relaxation rate (inverse of relaxation time) per unit concentration of the $\mathrm{CA}^{31}$

The relaxivity values for the different GOs are shown in Figure 5. The relaxivity curves for individual GO are shown in Supporting Information Figures S5 and S6. The values for each GO sample are provided in Supporting Information Table S4.
No substantial difference was found between the Tnull method and inversion recovery experiment on comparing the values obtained from the Tnull method and inversion recovery experiments with 16 delay periods.

When the $r_{1}$ and $r_{2}$ relaxivities are plotted for the GO series, we observe an irregular pattern in the variation of the relaxivities along the series. A distinguishable observation is that GO_80 has a considerably higher relaxivity. GO_50 has the lowest $r_{1}$ relaxivity followed by GO_0. GO_50 also has the lowest $r_{2}$ relaxivity.

On plotting the $r_{1}$ and $r_{2}$ relaxivities against the corresponding $\mathrm{Mn}^{2+}$ concentration (Figure 6a,b), we noted that samples having different $\mathrm{Mn}^{2+}$ concentrations exhibit same relaxivity and vice versa. GO_80 having the highest $\mathrm{Mn}^{2+}$ 

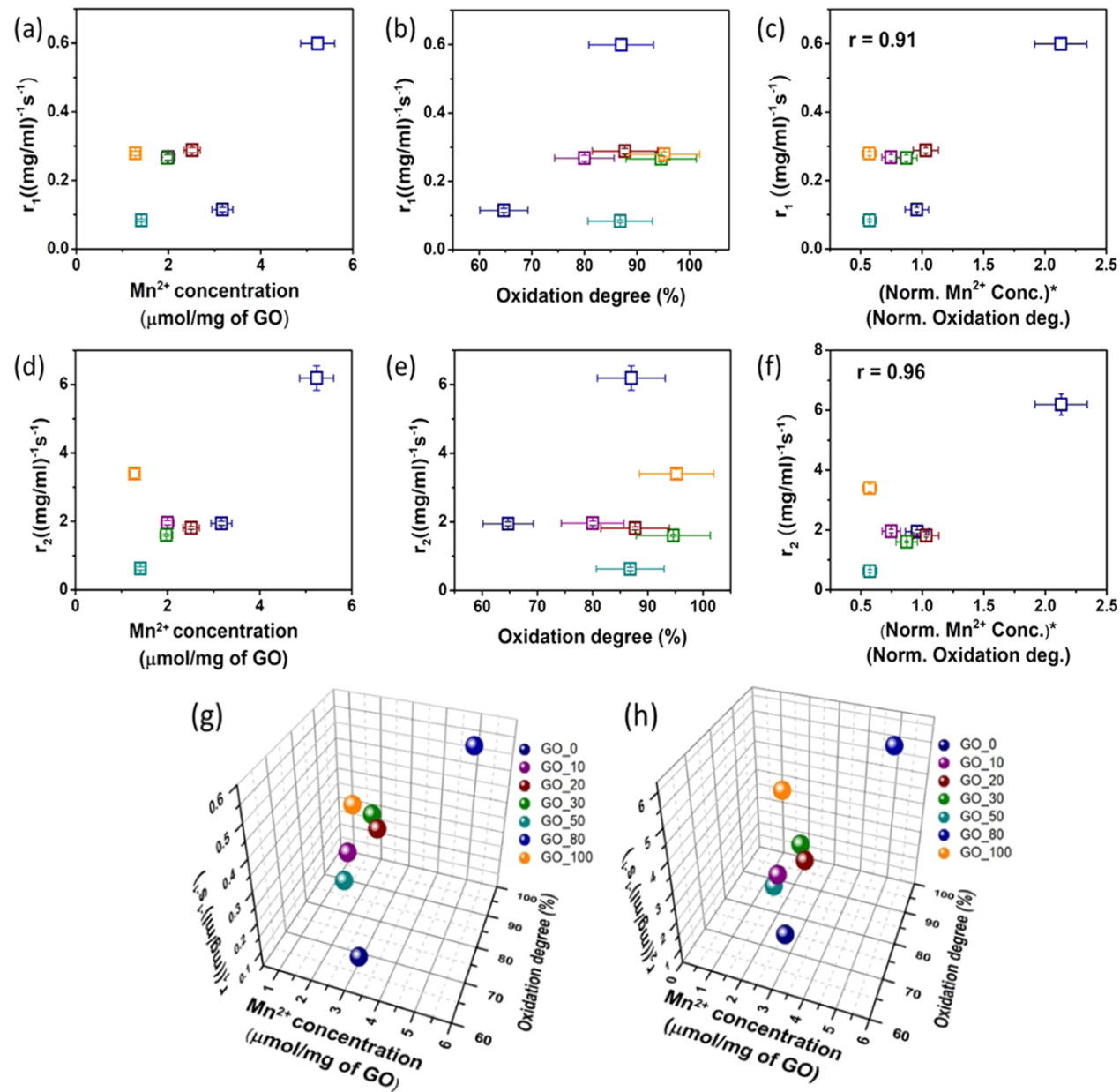

Figure 6. (a, d) Plots of $r_{1}$ and $r_{2}$ relaxivities as a function of $\mathrm{Mn}^{2+}$ concentration; (b, c) plots of $r_{1}$ and $r_{2}$ relaxivities as a function of oxidation degree; (e, f) plots of $r_{1}$ and $r_{2}$ relaxivities as a function of the product of mean-normalized $\mathrm{Mn}^{2+}$ concentration and oxidation degree to evaluate their correlation; $(\mathrm{g}, \mathrm{h})$ three-dimensional (3D) plots of $r_{1}$ and $r_{2}$ relaxivities with respect to $\mathrm{Mn}^{2+}$ concentration and oxidation degree. Color scheme used: blue square, GO_0; purple square, GO_10; red square, GO_20; green square, GO_30; peacock-green square, GO_50; blue square, GO_80; yellow square, $\mathrm{GO} \_100 . T_{1}$ and $T_{2}$ measurements were done at a magnetic field strength of $9.4 \mathrm{~T}$ at $298 \mathrm{~K}$.

concentration has the highest relaxivity; $\mathrm{GO}_{-} 0$ has higher $\mathrm{Mn}^{2+}$ than most of the other GO samples yet the lowest relaxivity values. GO_50 has similar concentrations of $\mathrm{Mn}^{2+}$ to GO_100 but much lower relaxivity than the latter. At the same time, on plotting $r_{1}$ and $r_{2}$ relaxivities against the corresponding oxidation degree for each GO (Figure 6d,e), we noted similar variations. Samples having different oxidation degrees exhibited similar relaxivities; GO_0 showed lower relaxivity values than other GOs; GO_100 having the highest oxidation degree had higher relaxivity values than other GOs, except GO_80. From the above observations, it can be said that both oxidation degree and $\mathrm{Mn}^{2+}$ concentration contribute to the relaxation behavior.

To decipher the combination of both these factors, the $\mathrm{Mn}^{2+}$ concentration and oxidation degree were normalized by their means and multiplied. The normalization was done by dividing the value of each $\mathrm{Mn}^{2+}$ concentration or the oxidation degree by their respective mean. The relaxivities were then plotted against this product (Figure 6c,f). The relaxivities were found to have a good correlation with the product of $\mathrm{Mn}^{2+}$ concentration and oxidation degree. From the 3D graphs (Figure 6g,h), we can see an overall increase in $r_{1}$ and $r_{2}$ relaxivities with respect to both the factors. Thus, in any given application, in addition to the concentration of $\mathrm{Mn}^{2+}$, it is important to take into account the oxidation degree of GO involved to tune its relaxivity. A variation of $\sim 30 \%$ in the oxidation degree in the GO shown translates to a 5-fold variation in $r_{1}$ and $r_{2}$ values.

The ability to change the relaxivity of GO was further evaluated for its contrast enhancement ability in MRI (Figure 7). Phantom images were acquired and processed using RadiAnt DICOM Viewer with the intensities measured for the chosen region of interest (ROI) (as illustrated in Figure S8). The area taken (area of the ROI) was $\sim 60 \%$ of the total area of the MR image of the cross section of the tube containing the sample, to avoid the peripheral artifacts. The $T_{1}$ contrast across the GO (Figure 7 ) varied from 81.1 to 570 , and the $T_{2}$ contrast varied from 49.4 to 235.9 (Figure S9). When we compare the $T_{1}$-weighted MR images (Figure 7a), we can clearly see better contrast in the case of GO_80 and very less contrast in the case of GO_50. Overall trends in the contrast along the GO series (Figure S9) with respect to blank are similar to their corresponding trend for $r_{1}$ and $r_{2}$ proton relaxivities.

Kinetic inertness of a material is a very important factor that should be accounted while designing an MRI contrast agent. Toward this end, the $r_{1}$ and $r_{2}$ values of the aqueous solutions were measured on day 1 and after 14 days of making the sample. This is shown in Figure S10. No significant changes in 
(a) Blank

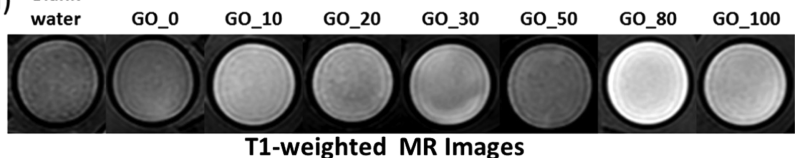

(b) Blank-

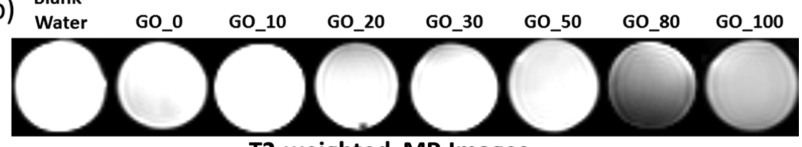

T2-weighted MR Images

Figure 7. Phantom MR images of GO suspensions in DI water acquired with a concentration of $500 \mu \mathrm{g} \mathrm{mL} L^{-1}$. (a) $T_{1}$-weighted MR images of GO suspensions and corresponding blank $\left(T_{1}\right.$-weighted $\mathrm{MR}$ image of water), $\mathrm{TR}=300 \mathrm{~ms}$, and $\mathrm{TE}=20 \mathrm{~ms}$; (b) $T_{2}$-weighted $\mathrm{MR}$ images of GO suspensions and corresponding blank $\left(T_{2}\right.$-weighted $M R$ image of water), $\mathrm{TE}=160 \mathrm{~ms}$, and $\mathrm{TR}=3000 \mathrm{~ms}$. MR imaging was performed at a magnetic field strength of $3 \mathrm{~T}$ at $296 \mathrm{~K}$.

the values could be observed, indicating that $\mathrm{Mn}^{2+}$ does not leach out of the GO with time. This also shows that $\mathrm{Mn}^{2+}$ ions are strongly embedded between the GO layers.

In recent years, a wide range of $T_{1}$ and $T_{2}$ contrast agents have been developed for MRI, such as gadolinium oxide nanoparticles, ${ }^{32,33}$ iron oxide nanoparticles, ${ }^{34,35}$ manganesebased nanoparticles, ${ }^{36-38}$ and metallic core-shell nanoparticles. ${ }^{39,40}$ Apart from the paramagnetic or superparamagnetic contrast agents, other types of CAs include particulate contrast agents (like fluorinated and nonfluorinated paramagnetic micelles or liposomes), diamagnetic hyperpolarization probes (gases and aerosols), metalloporphyrins, ${ }^{41,42}$ and carbon nanotubes. ${ }^{43}$ Wang et al. successfully synthesized water-dispersible $\mathrm{Gd}_{2} \mathrm{O}_{3} / \mathrm{GO}$ nanocomposites, which exhibited enhanced longitudinal relaxivity compared to previous Gadolinium-based MRI CAs. ${ }^{11}$ Venkatesha et. al. (2016) illustrated $\mathrm{CoFe}_{2} \mathrm{O}_{4}$-decorated $\mathrm{GO}$ as $T_{1}$ and $T_{2}$ CA for MRI. ${ }^{10}$ $\mathrm{Fe}_{3} \mathrm{O}_{4} / \mathrm{GO}$ composite has been exemplified as a $T_{2} \mathrm{CA} .{ }^{44}$ Peng et al. (2015) proposed a co-loading of GO with $\mathrm{Mn}$-doped $\mathrm{Fe}_{3} \mathrm{O}_{4}\left(T_{2}\right.$ agent) and $\mathrm{MnO}\left(T_{1}\right.$ agent $)$ magnetic nanoparticles, which exhibited $T_{1}$ and $T_{2}$ MRI contrast enhancement. $^{12}$

High relaxivity values can be achieved when GO is doped with paramagnetic and superparamagnetic nanoparticles. The probable ways in which GO plays a significant role in these complexes is by providing an optimal arrangement to the loaded nanoparticles, allowing them to generate high magnetic field gradients or restricting the movement of water molecules by their entrapment between the layers, thus increasing particle and proton interaction. In the present study, the interlayer spacing of GO is $\sim 10 \AA$ (based on XRD) and the size of water molecule is $\sim 2.75 \AA$. Thus, water can easily move between the layers, ${ }^{45}$ allowing interaction of the water molecules with the intercalated paramagnetic $\mathrm{Mn}$ ions.

To compare the relaxivity values of the GO series prepared in this study with previously reported paramagnetic and superparamagnetic MRI contrast agents, the $r_{1}$ and $r_{2}$ relaxivities of the GO samples were found by fitting the graphs of inverse of the longitudinal and transverse relaxation times vs the $\mathrm{Mn}^{2+}$ concentration of the samples (Table S5). The range of $r_{1}$ was $0.018-0.488 \mathrm{mM}^{-1} \mathrm{~s}^{-1}$, and that of $r_{2}$ was 0.193-4.159 $\mathrm{mM}^{-1} \mathrm{~s}^{-1}$ for the GO series. Table 1 exhibits a comparison of GO_80, which has the highest relaxivity values in the GO series with a few previously reported MRI contrast agents. GO_80 has an $r_{1}$ relaxivity value comparable to all of
Table 1. Comparison of $r_{1}$ and $r_{2}$ Relaxivities of GO 80 and Previously Reported Nanoparticle Systems for MRI

\begin{tabular}{|c|c|c|c|c|}
\hline sample & $\begin{array}{c}r_{1} \\
\left(\mathrm{mM}^{-1} \mathrm{~s}^{-1}\right)\end{array}$ & $\left(\mathrm{mM}^{r_{2}} \mathrm{~s}^{-1}\right)$ & $\begin{array}{l}\text { field } \\
(\mathrm{T})\end{array}$ & ref \\
\hline GO_80 & 0.49 & 4.16 & 9.4 & \\
\hline $\mathrm{HMnO} @ \mathrm{mSiO}_{2}$ & 0.99 & 11.02 & 11.7 & 46 \\
\hline MnO@PEG-phospholipid & 0.11 & 6.16 & 11.7 & 46 \\
\hline $\mathrm{MnO} @ \mathrm{mSiO}_{2}$ & 0.65 & 9.5 & 11.7 & 46 \\
\hline $\mathrm{MnO} @ \mathrm{dSiO}_{2}$ & 0.08 & 2.27 & 11.7 & 46 \\
\hline $\mathrm{ZnO} @ \mathrm{Gd}_{2} \mathrm{O}_{3}$ & 4.2 & 192 & 9.4 & 47 \\
\hline $\mathrm{ZnO} @ \mathrm{Gd}_{2} \mathrm{O}_{3} @ \mathrm{FA}$ & 0.15 & 36 & 9.4 & 47 \\
\hline PEG-PIONs/DOX complex & 1.4 & 179.07 & 9.4 & 48 \\
\hline
\end{tabular}

the contrast agents enlisted in Table 1 . The $r_{2}$ relaxivity value of GO 80 is comparable to the Mn-based contrast agents but lesser than ZnO@Gd2O3, ZnO@Gd2O3@FA, and PEGPIONs/DOX complex. However, the relaxivity values of GO_80 indicate that GO can act as both $T_{1}$ and $T_{2}$ MRI contrast agent for detecting hepatic lesions and scanning gastrointestinal tract. ${ }^{38}$ On plotting the $r_{1}$ relaxivity values of the GO samples at 3T, we observed better $r_{1}$ values than the values obtained at $9.4 \mathrm{~T}$, as shown in Figure $\mathrm{S} 11$. GO_80 is expected to confer a good $T_{1}$ contrast at $3 \mathrm{~T}$.

The contribution of GO to relaxivity, as revealed by the presented work, arises from the contribution of oxidation degree. The morphology of GO that we have been able to attain is due to the oxidization of extensively ball-milled graphitic precursor. Although we observed that $\mathrm{Mn}^{2+}$ intercalation is a stochastic process, a very important observation is that the defect density of GO 80 , prepared from BMG_80, which was ball-milled for $80 \mathrm{~h}$, is highest among all of the ball-milled graphitic precursors. This may be presumably the reason for the capturing of $\mathrm{Mn}^{2+}$ ions during the oxidation process. The GO_80 sample of the GO series clearly shows the effect of $\mathrm{Mn}^{2+}$, which allows comparison with GO with a low Mn content and a high oxidation degree and GO with a low oxidation degree and a high Mn content. The tuning of oxidation degree and architecture using ball milling and oxidizing of graphite can be strategically used to vary the $r_{1}$ and $r_{2}$ relaxivities. BMG 100 has more in-plane defects by virtue of sintering of smaller particles, causing these defects to be sites for surface functional groups, namely, hydroxyl and ketone groups.

\section{CONCLUSIONS}

The study provides new insights into the origin of proton relaxivity in graphene oxide and demonstrates the combined effect of oxidation degree and $\mathrm{Mn}^{2+}$ concentration on its relaxivity. The surface chemistry of GO can be modulated using the two-step methodology of ball milling graphite followed by oxidation. A variation of $\sim 30 \%$ in the oxidation degree of the GO is shown to translate to 5-fold variation in its relaxivity and image contrast values. This opens up new avenues for developing tunable GO-based contrast agents for different applications in magnetic resonance imaging.

\section{EXPERIMENTAL SECTION}

\subsection{Synthesis of GO with Varying Oxidation Degree.} The strategy for the synthesis of GO samples is depicted in Scheme 1. It involves a two-step methodology to prepare the GO samples; in the first step, the graphite powder is ball-milled for $X$ hours $(X=10,20,30,50,80,100)$, and then each 
Scheme 1. Schematic Illustration of the Two-Step Methodology for Engineering GO Chemistry Using Ball Milling of Graphite Followed by Oxidation to Graphene Oxide Using Tour's Method

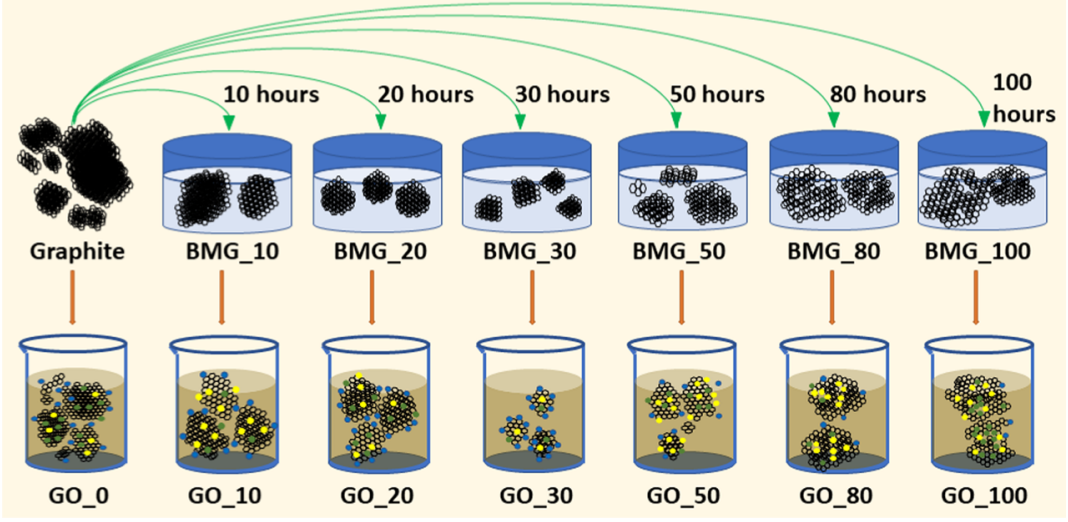

graphitic precursor is oxidized using Tour's method ${ }^{49}$ of oxidation, which is an improvement over the method described by Hummers and co-workers. ${ }^{50}$

The GO prepared from graphite is denoted as "GO_0". The ball-milled graphitic precursors are denoted as "BMG_X", and the corresponding graphene oxide samples are denoted as "GO $X$ ". Hence, the nomenclature for the precursor series is graphite, BMG_10, BMG_20, BMG_30, BMG_50, BMG_80, and $\mathrm{BMG}$ 100. Likewise, the $\mathrm{GO}$ series is $\mathrm{GO}_{-} 0, \mathrm{GO}_{-}^{-} 10$, GO 20, GO 30, GO 50, GO 80, and GO 100.

4.1.1. Planetary Baill Milling. In the planetary ball milling, a powder mixture is placed in a ball mill and exposed to highenergy collisions with stainless steel balls. Equal weight of graphite powder is taken in bowls and filled with hexane. Hexane is used as the ball milling medium as it has a low boiling point, and the ball-milled graphitic sample can be retrieved easily. The ball milling conditions used were: bowl speed, $700 \mathrm{rpm}$ and plate speed, $300 \mathrm{rpm}$; each cycle consisted of $15 \mathrm{~min}$ ON time and 8 min OFF time.

4.1.2. Tour's Method of Oxidation. A 1:6 w/w measure of graphitic precursor and potassium permanganate $\left(\mathrm{KMnO}_{4}\right)$ and a 9:1 v/v measure of sulfuric acid and orthophosphoric acid (total volume $=134 \mathrm{~mL}$ ) were taken in a beaker placed in an ice bath and continuously stirred for $12 \mathrm{~h}$ at $45^{\circ} \mathrm{C}$. The reaction mixture was slowly added to $140 \mathrm{~mL}$ of DI ice, followed by addition of $10 \mathrm{~mL}$ of hydrogen peroxide. The chrome yellow mixture was allowed to cool down, and $200 \mathrm{~mL}$ of deionized (DI) water was added. The final suspension was decanted for 2 days. The sediment was taken and washed thrice with DI water and twice with ethanol and then dried to obtain the graphene oxide powder.

4.2. Characterization of GO. 4.2.1. Raman Spectroscopy. Raman spectra were acquired using a LabRam HR with $532 \mathrm{~nm}$ wavelength. The samples were drop-dried on glass slides to form thin films. The spectra were recorded in the range of $1000-3000 \mathrm{~cm}^{-1}$. The peaks were then deconvoluted to acquire the defect density of the GO samples.

4.2.2. Electrophoretic Light Scattering (ELS). Zetasizer Nano (Malvern PanAnalytical) was used for the ELS study and to obtain $\zeta$-potentials for the GO samples. Measurements were performed at $25{ }^{\circ} \mathrm{C}$ and averaged from three sequential runs. $\mathrm{GO}$ suspension in water with a final concentration of $50 \mu \mathrm{g}$ $\mathrm{mL}^{-1}$ was used for the study.

4.2.3. Atomic Force Microscopy (AFM). The morphology of the GO samples was investigated using the NX-10 (Park
Systems) system. An Al back-coated $\mathrm{Si}$ probe (ACTA, AppNano, Inc.) with a resonance frequency of $300 \mathrm{kHz}$ and a nominal spring constant of $40 \mathrm{~N} \mathrm{~m}^{-1}$ was used. The GO samples were dispersed in water, bath-sonicated, drop-dried on Si wafers, and then gently rinsed with DI water. The images were obtained at a scan rate of $0.8 \mathrm{~Hz}$.

4.2.4. Solid-State NMR (ssNMR) Spectroscopy. An EXCII JEOL $400 \mathrm{MHz}$ spectrometer with a $4 \mathrm{~mm}$ magic angle spinning (MAS) probe was used for ssNMR spectroscopy. To acquire the ssNMR spectra, a ${ }^{13} \mathrm{C}$ single-pulse experiment was implemented with a relaxation delay period of $5 \mathrm{~s}$ between scans, 8192 scans, and a MAS speed of $10 \mathrm{KHz}$. The ssNMR spectra were obtained to determine the extent of oxidation in the GO samples. The intensity (area) of peaks pertaining to unreacted graphite and that of the carbon backbone peaks corresponding to $\mathrm{GO}$ were used for calculation of oxidation degrees of GO along with the data from XPS.

4.2.5. X-ray Photoelectron Spectroscopy (XPS). The elemental composition of GO was determined using a Kratos $\mathrm{X}$-ray photoelectron spectrometer with $\mathrm{Al} \mathrm{K} \alpha \mathrm{X}$-ray source having an excitation energy of $1486.6 \mathrm{eV}$. For each set of sample, wide spectra and high-resolution $C$ 1s spectra were recorded. The ratio of nonaromatic carbon to total carbon (sum of aromatic carbon and nonaromatic carbon) was calculated by deconvolution of the high-resolution $\mathrm{C} 1 \mathrm{~s}$ spectra. This information was used to calculate the oxidation degree of the samples of interest.

From the deconvolution of high-resolution $\mathrm{C}$ 1s scans of the GO acquired using XPS, the percentage of aromatic and nonaromatic carbon was measured, and using solid-state NMR spectroscopy (ssNMR), the amount of oxidized graphite could be measured. These two parameters were then used to calculate the oxidation degree using the formula ${ }^{51}$

$$
\begin{aligned}
& \text { oxidation degree }(\mathrm{OD}) \text { of } \mathrm{GO} \\
& \begin{aligned}
= & {\left[I_{\text {nonaromatic }} /\left(I_{\text {aromatic }}+I_{\text {nonaromatic }}\right)\right] } \\
& \times\left[I_{\mathrm{GO}} /\left(I_{\text {graphite }}+I_{\mathrm{GO}}\right)\right] \times 100
\end{aligned}
\end{aligned}
$$

where $I_{\text {aromatic }}$ and $I_{\text {nonaromatic }}$ are calculated from XPS, and $I_{\text {graphite }}$ and $I_{\mathrm{GO}}$ are calculated from the total area under the peaks in the ssNMR spectrum corresponding to unreacted graphite and GO.

4.2.6. Atomic Absorption Spectroscopy (AAS). A Thermo Electron Corporation M-Series machine was used for atomic 
absorption spectroscopy (AAS). Quantitative elemental estimation of $\mathrm{Fe}^{2+}$ and $\mathrm{Fe}^{3+}$ was obtained. Standard solutions of iron were prepared using ferrous ammonium sulfate (FAS) salt in double-distilled water; $1-5 \mathrm{ppm}$ iron solutions were prepared by dilution. The GO samples with known weight were dissolved using nitric acid and then diluted with distilled water. The solutions were filtered using a microfilter to remove undissolved residue. The sample solution absorbance was recorded using an AAS instrument along with standard solutions.

4.2.7. Electron Paramagnetic Resonance (EPR) Spectroscopy. JES-X3 SERIES A SYSTEM was used for electron paramagnetic resonance (EPR) spectroscopy. All of the EPR spectra were acquired at a temperature of $298 \mathrm{~K}$ under identical conditions. The operating frequency was $\sim 9.45 \mathrm{GHz}$ with a modulation frequency of $100 \mathrm{kHz}$ and $0.99 \mathrm{~mW}$ power. Calibration was done using a solid sample of diphenyl picrylhydrazyl (DPPH). The field center was $336 \mathrm{mT}$ with a sweep width of $50 \mathrm{mT}$ for acquisition of the EPR signal. For quantification, a standard was prepared with $\mathrm{MnCl}_{2}$ in the $\mathrm{KCl}$ matrix with a $10 \%$ molar ratio. $\mathrm{MnCl}_{2}$ and $\mathrm{KCl}$ were dissolved in water and mixed well and then lyophilized to obtain a uniformly distributed solute in $\mathrm{KCl}$. Three samples of the standard were taken to measure the average spin concentration of the standard. An equal amount of GO samples was taken to quantify the respective average spin concentration, and the $\mathrm{Mn}^{2+}$ concentration was calculated. The EPR spectra were double-integrated to obtain the intensities of the signals, and the spins for the standard sample and the samples were calculated.

4.3. Relaxivity Measurements. 4.3.1. $r_{1}$ Relaxivity. The $r 1$ relaxivity was obtained from spin-lattice relaxation time $\left(T_{1}\right)$ measurements at $298 \mathrm{~K}$ on a Bruker Avance NMR spectrometer operating at a ${ }^{1} \mathrm{H}$ resonance frequency of 400 $\mathrm{MHz}$. The sample was prepared by weighing different amounts of GO and preparing eight dilutions in DI water. For each concentration of the sample, an independent experiment was performed. The $T_{1}$ relaxation time was measured by a onedimensional inversion recovery NMR experiment. ${ }^{52}$ In this experiment, a $180^{\circ}$ radio frequency (r.f.) pulse inverts the bulk magnetization from its equilibrium position along the $+z$-axis to the $-z$-axis, followed by a delay. During this delay period, due to $T_{1}$ relaxation along the longitudinal axis, the magnetization recovers toward the equilibrium state. A $90^{\circ}$ r.f. pulse is applied after the delay period to create a transverse magnetization. Following this, the acquisition of the signal is carried out. In each experiment, the value of the delay period is varied, ranging from 0 to $2 \mathrm{~s}$. The delay period at which the recovery of the magnetization from $-z$-axis goes through a null was considered as " $T_{\text {null }}$ ", and $T_{1}$ was calculated using the formula $T_{1}=T_{\text {null }} / \ln 2,{ }^{52}$ and the longitudinal proton relaxation rate $R_{1}$ was calculated by taking the inverse of $T_{1}$.

4.3.2. $r_{2}$ Relaxivity. The $r_{2}$ relaxivity was obtained from spin-spin relaxation time $\left(T_{2}\right)$ measurements at $298 \mathrm{~K}$ on a Bruker Avance NMR spectrometer operating at a ${ }^{1} \mathrm{H}$ resonance frequency of $400 \mathrm{MHz}$. The measurements were carried out using the one-dimensional Carr-Purcell-Meiboom-Gill sequence (CPMG) experiment. ${ }^{53}$ In this experiment, a $90^{\circ}$ r.f. pulse is applied to create the transverse magnetization, followed by a spin-echo period, which consists of a [delay$180^{\circ}$ r.f. pulse-delay] block during the magnetization de-phases in the transverse plane due to $T_{2}$ relaxation. The spin-echo block is repeated $n$ times in sequence to obtain a total desired delay period for allowing the magnetization to relax. After the repetition of the spin-echo block for a desired number of times, the signal is acquired. A series of spectra are acquired with different relaxation delays (by varying the number of repetitions of the spin-echo block). $R_{2}$ is obtained by fitting the measured intensities to the relaxation time periods (decreasing monoexponential graph) using the formula $y=$ $y_{0}+A_{1} \times \exp \left(-\left(x-x_{0}\right) \times R_{2}\right)$, to obtain the transverse proton relaxation rate $R_{2}\left(1 / T_{2}\right)$, where $y$ is the intensity, $y_{0}$ is the $y$ intercept, $A_{1}$ is the amplitude, $x$ is the total evolution time, and $x_{0}$ is $x$-offset. ${ }^{54}$ The same sample as used for $r_{1}$ relaxivity measurements was used for $T_{2}$ measurements. The following relaxation delays were used: $6,30,60,90,120,150,180,210$, $240,270,300,600,900,1200,1500,1800,2100,2400,2700$, $3000 \mathrm{~ms}$

4.4. Magnetic Resonance Imaging (MRI). The phantom imaging study was performed on a $3 \mathrm{~T}$ clinical MRI scanner (Ingenia, Philips, the Netherlands) using a 32-channel transmit-received head coil at $296 \mathrm{~K}$. All of the phantom data were acquired in axial orientation using $T_{1^{-}}$and $T_{2^{-}}$ weighted imaging sequences. Fixed echo time ( $\mathrm{TE}=20 \mathrm{~ms}$ ) and repetition time ( $\mathrm{TR}=300 \mathrm{~ms}$ ) were used to obtain the $T_{1}$ weighted images for various GO suspensions. Similarly, for obtaining $T_{2}$-weighted images, the fixed echo time was $160 \mathrm{~ms}$ and the repetition time was $3000 \mathrm{~ms}$. The other acquisition parameters were slice thickness $=6 \mathrm{~mm}, \mathrm{FOV}=200 \times 200$, and matrix size $=128 \times 128$. The total acquisition time was $\sim 45 \mathrm{~min} /$ phantom study. For obtaining the $r_{1}$ relaxivity, $T_{1}$ images for all samples with concentrations varying from 100 to $500 \mu \mathrm{g} \mathrm{mL}^{-1}$ were acquired at $\mathrm{TR}=300,600,1000,1500$, 2000, 3000. 5000, and $7000 \mathrm{~ms}$. Information was extracted from the MR images, and the intensities were plotted against the TR values and fitted with exponential function to obtain the $T_{1}$ relaxation constants. Inverse values of these $T_{1}$ values were plotted against respective concentration for each sample to determine its $r_{1}$ relaxivity value. The $T_{2}$ CALC experiment was implemented with $\mathrm{TR}=3000 \mathrm{~ms}$ and $\mathrm{TE}=20-160 \mathrm{~ms}$ to find the $T_{2}$ relaxation times of the samples, and the $r_{2}$ relaxivity values for the GO samples were calculated.

4.5. Data Analysis. Data plotting and analysis were done using the OriginPro 8.5.1 and GraphPad Prism 5 softwares. Microsoft Excel has also been used for data management and statistical analysis.

\section{ASSOCIATED CONTENT}

\section{Supporting Information}

The Supporting Information is available free of charge at https://pubs.acs.org/doi/10.1021/acsomega.0c02220.

AFM images, XPS, Raman, and solid-state NMR data of GO samples; $r_{1}$ and $r_{2}$ relaxivity graphs; view of the phantom with samples; variation of $T_{1}$ and $T_{2}$ contrast for $T_{1}$-weighted and $T_{2}$-weighted MRI images, respectively, along the GO series; and $r_{1}$ and $r_{2}$ relaxivities at different time durations (PDF)

\section{AUTHOR INFORMATION}

\section{Corresponding Author}

Zinia Mohanta - Centre for BioSystems Science and Engineering, Indian Institute of Science, Bengaluru 560012, India; 다이.org/0000-0001-8921-044X; Email: zmohanta17@ gmail.com, ziniamohanta@iisc.ac.in 


\section{Authors}

Sumana K. Gaonkar - Nuclear Magnetic Resonance Research Centre, Indian Institute of Science, Bengaluru 560012, India

Manoj Kumar - Department of Neuroimaging and Interventional Radiology, National Institute of Mental Health and Neurosciences, Bengaluru 560029, India

Jitender Saini - Department of Neuroimaging and Interventional Radiology, National Institute of Mental Health and Neurosciences, Bengaluru 560029, India

Vivek Tiwari - Centre for Brain Research, Indian Institute of Science, Bengaluru 560012, India

Chandan Srivastava - Department of Materials Engineering, Indian Institute of Science, Bengaluru 560012, India; (1) orcid.org/0000-0001-9732-4842

Hanudatta S. Atreya - Nuclear Magnetic Resonance Research Centre, Indian Institute of Science, Bengaluru 560012, India; (1) orcid.org/0000-0003-0200-7372

Complete contact information is available at:

https://pubs.acs.org/10.1021/acsomega.0c02220

\section{Author Contributions}

The manuscript was written through contributions of all authors. All authors have given approval to the final version of the manuscript.

\section{Funding}

This work was supported by grants received from Department of Science and Technology (IR/S0/LU-007/2010), Govt. of India.

\section{Notes}

The authors declare no competing financial interest.

\section{ACKNOWLEDGMENTS}

The authors thank the NMR Research Centre, Department of Materials Engineering, Department of Biosystems Science and Engineering and Centre for Nanoscience at the Indian Institute of Science for providing the different NMR and nanomaterial characterization facilities, respectively. H.S.A. acknowledges research grant from Department of Science and Technology (IR/S0/LU-007/2010). The MRI facility at Centre for Brain Research, IISc, Bangalore, and National Institute of Mental Health and Neurosciences (NIMHANS), Bangalore, is gratefully acknowledged. The authors acknowledge the EPR and NMR Facility, Central Leather Research Institute, Chennai, for help in EPR data acquisition.

\section{REFERENCES}

(1) Sun, X.; Liu, Z.; Welsher, K.; Robinson, J. T.; Goodwin, A.; Zaric, S.; Dai, H. Nano-Graphene Oxide for Cellular Imaging and Drug Delivery. Nano Res. 2008, 1, 203-212.

(2) Loh, K. P.; Bao, Q.; Eda, G.; Chhowalla, M. Graphene Oxide as a Chemically Tunable Platform for Optical Applications. Nat. Chem. 2010, 2, 1015-1024.

(3) Madadrang, C. J.; Kim, H. Y.; Gao, G.; Wang, N.; Zhu, J.; Feng, H.; Gorring, M.; Kasner, M. L.; Hou, S. Adsorption Behavior of EDTA-Graphene Oxide for Pb (II) Removal. ACS Appl. Mater. Interfaces 2012, 4, 1186-1193.

(4) Yang, D.; Velamakanni, A.; Bozoklu, G.; Park, S.; Stoller, M.; Piner, R. D.; Stankovich, S.; Jung, I.; Field, D. A.; Ventrice, C. A.; Ruoff, R. S. Chemical Analysis of Graphene Oxide Films after Heat and Chemical Treatments by X-Ray Photoelectron and Micro-Raman Spectroscopy. Carbon 2009, 47, 145-152.

(5) Mohanta, Z.; Atreya, H. S.; Srivastava, C. Correlation between Defect Density in Mechanically Milled Graphite and Total Oxygen
Content of Graphene Oxide Produced from Oxidizing the Milled Graphite. Sci. Rep. 2018, 8, No. 15773.

(6) Li, R.; Guiney, L. M.; Chang, C. H.; Mansukhani, N. D.; Ji, Z.; Wang, X.; Liao, Y.-P.; Jiang, W.; Sun, B.; Hersam, M. C.; Nel, A. E.; Xia, T. Surface Oxidation of Graphene Oxide Determines Membrane Damage, Lipid Peroxidation, and Cytotoxicity in Macrophages in a Pulmonary Toxicity Model. ACS Nano 2018, 12, 1390-1402.

(7) Malik, S. A.; Mohanta, Z.; Srivastava, C.; Atreya, H. S. Modulation of Protein-Graphene Oxide Interactions with Varying Degrees of Oxidation. Nanoscale Adv. 2020, 2, 1904-1912.

(8) Guerrero-Contreras, J.; Caballero-Briones, F. Graphene Oxide Powders with Different Oxidation Degree, Prepared by Synthesis Variations of the Hummers Method. Mater. Chem. Phys. 2015, 153, 209-220.

(9) Compton, O. C.; Jain, B.; Dikin, D. A.; Abouimrane, A.; Amine, K.; Nguyen, S. T. Chemically Active Reduced Graphene Oxide with Tunable C/O Ratios. ACS Nano 2011, 5, 4380-4391.

(10) Venkatesha, N.; Poojar, P.; Ashwini, R.; Qurishi, Y.; Geethanath, S.; Srivastava, C. Ultrafine Graphene Oxide-CoFe 2 O 4 Nanoparticle Composite as $\mathrm{T} 1$ and $\mathrm{T} 2$ Contrast Agent for Magnetic Resonance Imaging. RSC Adv. 2016, 6, 17423-17429.

(11) Wang, F.; Peng, E.; Zheng, B.; Li, S. F. Y.; Xue, J. M. Synthesis of Water-Dispersible Gd2O3/GO Nanocomposites with Enhanced MRI T1 Relaxivity. J. Phys. Chem. C 2015, 119, 23735-23742.

(12) Peng, E.; Wang, F.; Tan, S.; Zheng, B.; Li, S. F. Y.; Xue, J. M. Tailoring a Two-Dimensional Graphene Oxide Surface: Dual T 1 and T 2 MRI Contrast Agent Materials. J. Mater. Chem. B 2015, 3, 56785682.

(13) Roguin, A.; Schwitter, J.; Vahlhaus, C.; Lombardi, M.; Brugada, J.; Vardas, P.; Auricchio, A.; Priori, S.; Sommer, T. Magnetic Resonance Imaging in Individuals with Cardiovascular Implantable Electronic Devices †. EP Eur. 2008, 10, 336-346.

(14) Hashemi, R. H.; Bradley, W. G.; Lisanti, C. J. MRI: The Basics: The Basics; Lippincott Williams \& Wilkins, 2012.

(15) O'Connor, E. E.; Dixon, L. B.; Peabody, T.; Stacy, G. S. MRI of Cystic and Soft-Tissue Masses of the Shoulder Joint. Am. J. Roentgenol. 2004, 183, 39-47.

(16) Ladd, M. E.; Bachert, P.; Meyerspeer, M.; Moser, E.; Nagel, A. M.; Norris, D. G.; Schmitter, S.; Speck, O.; Straub, S.; Zaiss, M. Pros and Cons of Ultra-High-Field MRI/MRS for Human Application. Prog. Nucl. Magn. Reson. Spectrosc. 2018, 109, 1-50.

(17) Mastrogiacomo, S.; Dou, W.; Jansen, J. A.; Walboomers, X. F. Magnetic Resonance Imaging of Hard Tissues and Hard Tissue Engineered Bio-Substitutes. Mol. Imaging Biol. 2019, 21, 1003-1019.

(18) Laurent, V.; Trausch, G.; Bruot, O.; Olivier, P.; Felblinger, J.; Régent, D. Comparative Study of Two Whole-Body Imaging Techniques in the Case of Melanoma Metastases: Advantages of Multi-Contrast MRI Examination Including a Diffusion-Weighted Sequence in Comparison with PET-CT. Eur. J. Radiol. 2010, 75, 376-383.

(19) Tyler, D. J. Cardiovascular Applications of Hyperpolarized MRI. Curr. Cardiovasc. Imaging Rep. 2011, 4, 108-115.

(20) Na, H. Bin.; Song, I. C.; Hyeon, T. Inorganic Nanoparticles for MRI Contrast Agents. Adv. Mater. 2009, 21, 2133-2148.

(21) Roberts, T. P. L.; Chuang, N.; Roberts, H. C. Neuroimaging: Do We Really Need New Contrast Agents for MRI? Eur. J. Radiol. 2000, 34, 166-178.

(22) Jacques, V.; Dumas, S.; Sun, W.-C.; Troughton, J. S.; Greenfield, M. T.; Caravan, P. High-Relaxivity Magnetic Resonance Imaging Contrast Agents. Part 2. Optimization of Inner- and SecondSphere Relaxivity. Invest. Radiol. 2010, 45, 613-624.

(23) Paratala, B. S.; Jacobson, B. D.; Kanakia, S.; Francis, L. D.; Sitharaman, B. Physicochemical Characterization, and Relaxometry Studies of Micro-Graphite Oxide, Graphene Nanoplatelets, and Nanoribbons. PLoS One 2012, 7, e38185.

(24) Panich, A. M.; Shames, A. I.; Sergeev, N. A. Paramagnetic Impurities in Graphene Oxide. Appl. Magn. Reson. 2013, 44, 107-116.

(25) Shen, T. D.; Ge, W. Q.; Wang, K. Y.; Quan, M. X.; Wang, J. T.; Wei, W. D.; Koch, C. C. Structural Disorder and Phase Trans- 
formation in Graphite Produced by Ball Milling. Nanostruct. Mater. 1996, 7, 393-399.

(26) Wang, Y.; Gao, Y.; Li, Y.; Zhang, C.; Huang, X.; Zhai, W. Effect of Milling Time on Microstructure and Mechanical Properties of $\mathrm{Cu}-$ Ni-Graphite Composites. Mater. Res. Express 2017, 4, No. 096506.

(27) Xing, T.; Li, L. H.; Hou, L.; Hu, X.; Zhou, S.; Peter, R.; Petravic, M.; Chen, Y. Disorder in Ball-Milled Graphite Revealed by Raman Spectroscopy. Carbon 2013, 57, 515-519.

(28) Scarpulla, M. A.; Dubon, O. D.; Yu, K. M.; Monteiro, O.; Pillai, M. R.; Aziz, M. J.; Ridgway, M. C. Ferromagnetic Ga1-xMnxAs Produced by Ion Implantation and Pulsed-Laser Melting. Appl. Phys. Lett. 2003, 82, 1251-1253.

(29) Panich, A. M.; Shames, A. I.; Aleksenskii, A. E.; Dideikin, A. Magnetic Resonance Evidence of Manganese-Graphene Complexes in Reduced Graphene Oxide. Solid State Commun. 2012, 152, 466468.

(30) Shokrollahi, H. Contrast Agents for MRI. Mater. Sci. Eng.: C 2013, 33, 4485-4497.

(31) Lohrke, J.; Frenzel, T.; Endrikat, J.; Alves, F. C.; Grist, T. M.; Law, M.; Lee, J. M.; Leiner, T.; Li, K.-C.; Nikolaou, K.; Prince, M. R.; Schild, H. H.; Weinreb, J. C.; Yoshikawa, K.; Pietsch, H. 25 Years of Contrast-Enhanced MRI: Developments, Current Challenges and Future Perspectives. Adv. Ther. 2016, 33, 1-28.

(32) Roux, S.; Faure, A. C.; Celine, M.; Riviere, C.; Bridot, J.-L.; Mutelet, B.; Marquette, C. A.; Josser, V.; Le Duc, G.; Pape, L.; et al. Multifunctional Gadolinium Oxide Nanoparticles: Towards ImageGuided Therapy. Imaging Med. 2010, 2, 211-223.

(33) Kryza, D.; Taleb, J.; Janier, M.; Marmuse, L.; Miladi, I.; Bonazza, P.; Louis, C.; Perriat, P.; Roux, S.; Tillement, O.; Billotey, C. Biodistribution Study of Nanometric Hybrid Gadolinium Oxide Particles as a Multimodal SPECT/MR/Optical Imaging and Theragnostic Agent. Bioconjugate Chem. 2011, 22, 1145-1152.

(34) Wang, Y.-X. J. Superparamagnetic Iron Oxide Based MRI Contrast Agents: Current Status of Clinical Application. Quant. Imaging Med. Surg. 2011, 1, 35-40.

(35) Smolensky, E. D.; Park, H.-Y. E.; Berquó, T. S.; Pierre, V. C. Surface Functionalization of Magnetic Iron Oxide Nanoparticles for MRI Applications - Effect of Anchoring Group and Ligand Exchange Protocol. Contrast Media Mol. Imaging 2011, 6, 189-199.

(36) Chen, Y.; Chen, H.; Zhang, S.; Chen, F.; Sun, S.; He, Q.; Ma, M.; Wang, X.; Wu, H.; Zhang, L.; Zhang, L.; Shi, J. Structure-Property Relationships in Manganese Oxide - Mesoporous Silica Nanoparticles Used for T1-Weighted MRI and Simultaneous Anti-Cancer Drug Delivery. Biomaterials 2012, 33, 2388-2398.

(37) Shapiro, E. M.; Koretsky, A. P. Convertible Manganese Contrast for Molecular and Cellular MRI. Magn. Reson. Med. 2008, 60, 265-269.

(38) Xing, R.; Zhang, F.; Xie, J.; Aronova, M.; Zhang, G.; Guo, N.; Huang, X.; Sun, X.; Liu, G.; Bryant, L. H.; et al. Polyaspartic Acid Coated Manganese Oxide Nanoparticles for Efficient Liver MRI. Nanoscale 2011, 3, 4943-4945.

(39) Venkatesha, N.; Qurishi, Y.; Atreya, H. S.; Srivastava, C. Effect of Core-Shell Nanoparticle Geometry on the Enhancement of the Proton Relaxivity Value in a Nuclear Magnetic Resonance Experiment. RSC Adv. 2016, 6, 64605-64610.

(40) Venkatesha, N.; Pudakalakatti, S. M.; Qurishi, Y.; Atreya, H. S.; Srivastava, C. $\mathrm{MnFe}_{2} \mathrm{O}_{4}-\mathrm{Fe}_{3} \mathrm{O}_{4}$ Core-Shell Nanoparticles as a Potential Contrast Agent for Magnetic Resonance Imaging. RSC Adv. 2015, 5, 97807-97815.

(41) Estelrich, J.; Sánchez-Martín, M. J.; Busquets, M. A. Nanoparticles in Magnetic Resonance Imaging: From Simple to Dual Contrast Agents. Int. J. Nanomed. 2015, 10, 1727-1741.

(42) Palekar, R. U.; Jallouk, A. P.; Lanza, G. M.; Pan, H.; Wickline, S. A. Molecular Imaging of Atherosclerosis with Nanoparticle-Based Fluorinated MRI Contrast Agents. Nanomedicine 2015, 10, 18171832.

(43) Vittorio, O.; Duce, S. L.; Pietrabissa, A.; Cuschieri, A. Multiwall Carbon Nanotubes as $\{$ MRI $\}$ Contrast Agents for Tracking Stem Cells. Nanotechnology 2011, 22, 95706.
(44) Zhou, C.; Wu, H.; Wang, M.; Huang, C.; Yang, D.; Jia, N. Functionalized Graphene Oxide/Fe3O4 Hybrids for Cellular Magnetic Resonance Imaging and Fluorescence Labeling. Mater. Sci. Eng.: C 2017, 78, 817-825.

(45) Cho, Y. H.; Kim, H. W.; Lee, H. D.; Shin, J. E.; Yoo, B. M.; Park, H. B. Water and Ion Sorption, Diffusion, and Transport in Graphene Oxide Membranes Revisited. J. Membr. Sci. 2017, 544, $425-435$.

(46) Kim, T.; Momin, E.; Choi, J.; Yuan, K.; Zaidi, H.; Kim, J.; Park, M.; Lee, N.; McMahon, M. T.; Quinones-Hinojosa, A.; Bulte, J. W. M.; Hyeon, T.; Gilad, A. A. Mesoporous Silica-Coated Hollow Manganese Oxide Nanoparticles as Positive T1 Contrast Agents for Labeling and MRI Tracking of Adipose-Derived Mesenchymal Stem Cells. J. Am. Chem. Soc. 2011, 133, 2955-2961.

(47) Babayevska, N.; Florczak, P.; Woźniak-Budych, M.; Jarek, M.; Nowaczyk, G.; Zalewski, T.; Jurga, S. Functionalized Multimodal ZnO@Gd2O3 Nanosystems to Use as Perspective Contrast Agent for MRI. Appl. Surf. Sci. 2017, 404, 129-137.

(48) Hałupka-Bryl, M.; Bednarowicz, M.; Dobosz, B.; Krzyminiewski, R.; Zalewski, T.; Wereszczyńska, B.; Nowaczyk, G.; Jarek, M.; Nagasaki, Y. Doxorubicin Loaded PEG-b-Poly(4-Vinylbenzylphosphonate) Coated Magnetic Iron Oxide Nanoparticles for Targeted Drug Delivery. J. Magn. Magn. Mater. 2015, 384, 320-327. (49) Marcano, D. C.; Kosynkin, D. V.; Berlin, J. M.; Sinitskii, A.; Sun, Z.; Slesarev, A.; Alemany, L. B.; Lu, W.; Tour, J. M. Improved Synthesis of Graphene Oxide. ACS Nano 2010, 4, 4806-4814.

(50) Hummers, W. S.; Offeman, R. E. Preparation of Graphitic Oxide. J. Am. Chem. Soc. 1958, 80, 1339.

(51) Yan, H.; Tao, X.; Yang, Z.; Li, K.; Yang, H.; Li, A.; Cheng, R. Effects of the Oxidation Degree of Graphene Oxide on the Adsorption of Methylene Blue. J. Hazard. Mater. 2014, 268, 191-198.

(52) Bain, A. D. The Choice of Parameters in an NMR Experiment. Application to the Inversion-Recovery T1 Method. J. Magn. Reson. 1990, 89, 153-160.

(53) Blümich, B. Introduction to Compact NMR: A Review of Methods. TrAC Trends Anal. Chem. 2016, 83, 2-11.

(54) Otto, R.; Ferguson, M. R.; Marro, K.; Grinstead, J. W.; Friedman, S. D. Limitations of Using Logarithmic Transformation and Linear Fitting to Estimate Relaxation Rates in Iron-Loaded Liver. Pediatr. Radiol. 2011, 41, 1259-1265. 